\title{
Nonexistence of triples of nonisomorphic connected graphs with isomorphic connected $P_{3}$-graphs *
}

\author{
Xueliang Li and Yan Liu \\ Center for Combinatorics and LPMC \\ Nankai University, Tianjin 300071, China \\ lxl@nankai.edu.cn, liuyanday@yahoo.com.cn
}

Submitted: Aug 21, 2006; Accepted: Feb 3, 2008; Published: Feb 11, 2008

Mathematics Subject Classifications: 05C60, 05C75

\begin{abstract}
In the paper "Broersma and Hoede, Path graphs, J. Graph Theory 13 (1989) 427-444", the authors asked a problem whether there is a triple of mutually nonisomorphic connected graphs which have an isomorphic connected $P_{3}$-graph. In this paper, we show that there is no such triple, and thus completely solve this problem.
\end{abstract}

Keywords: path graph, connected, isomorphism

\section{Introduction}

Broersma and Hoede [3] generalized the concept of line graphs to that of path graphs by defining adjacency as follows. Let $k$ be a positive integer, and $P_{k}$ and $C_{k}$ denote a path and a cycle with $k$ vertices, respectively. Let $\Pi_{k}(G)$ be the set of all $P_{k}$ 's in $G$. The path graph $P_{k}(G)$ of $G$ is a graph with vertex set $\Pi_{k}(G)$ in which two $P_{k}$ 's are adjacent whenever their union is a path $P_{k+1}$ or a cycle $C_{k}$. Broersma and Hoede got many results on $P_{3}$-graphs and, in particular, described two infinite classes of pairs of nonisomorphic connected graphs which have isomorphic connected $P_{3}$-graphs. They also raised a number of unsolved problems or questions, most of which have been solved in the intervening years. Only the following one remains unanswered.

Problem. Does there exist a triple of mutually nonisomorphic connected graphs which have an isomorphic connected $P_{3}$-graph ?

\footnotetext{
*Research supported by PCSIRT, NSFC and the "973" program.
} 
For $k=2$, i.e., line graphs, from Whitney's result (see [4]) it is not difficult to see that the problem has a negative answer. In [5] the authors showed that for $k \geq 4$ there are not only triples of but also arbitrarily many mutually nonisomorphic connected graphs with isomorphic connected $P_{k}$-graphs. Interestingly, however, we will show in this paper that for $k=3$ there does not exist any triple of mutually nonisomorphic connected graphs with an isomorphic connected $P_{3}$-graph; just like the case for $k=2$ but very different from the case for $k \geq 4$. Note that if one drops the connectedness of the original graph or its $P_{3}$-graph, then it is easy to find arbitrarily many mutually nonisomorphic graphs with an isomorphic $P_{3}$-graph.

\section{Preliminaries}

All graphs in this paper are undirected, finite and simple. We follow the terminology and notations used in $[1,2]$. If $\sigma$ is an isomorphism from $G$ to $H$, then $\sigma$ induces a $P_{k}$-isomorphism $\sigma^{*}$ from $G$ to $H$, where $\sigma^{*}\left(a_{1} a_{2} \cdots a_{k}\right)=\sigma\left(a_{1}\right) \sigma\left(a_{2}\right) \cdots \sigma\left(a_{k}\right)$ for all $a_{1} a_{2} \cdots a_{k} \in \Pi_{k}(G)$. A $P_{k}$-isomorphism $\tau$ is induced if $\tau=\sigma^{*}$ for some isomorphism $\sigma$. If $\tau_{i}$ is a $P_{k}$-isomorphism from $G_{i}$ to $H_{i}$ for $i=1$ and 2 , then we say that $\tau_{1}$ and $\tau_{2}$ are equivalent if there are isomorphisms $\sigma$ and $\rho$ from $G_{1}$ to $G_{2}$ and $H_{1}$ to $H_{2}$, respectively, such that $\tau_{1}=\left(\rho^{*}\right)^{-1} \circ \tau_{2} \circ \sigma^{*}$.

A vertex of degree 1 is called terminal, and an edge is terminal if it has a terminal end. Define an $i$-thorn to be a $P_{3}$ with exactly $i(i=1$ or 2$)$ terminal ends in $G$. Let $T_{i}(G)$ be the set of $i$-thorns in $G$. A $P_{3}$ in $G$ is called terminal if it has degree 1 in $P_{3}(G)$.

We say that two $P_{3}$-isomorphisms $\tau_{i}$ from $G_{i}$ to $H_{i}$ for $i=1$ and 2 , are $T$-related if $(i)$ $G_{1}$ and $G_{2}$ differ only in their star components, so do $H_{1}$ and $H_{2} ;(i i)\left|T_{2}\left(G_{1}\right)\right|=\left|T_{2}\left(G_{2}\right)\right|$; and $(i i i) \tau_{1}(\alpha)=\tau_{2}(\alpha)$ for every $\alpha \in \Pi_{3}\left(G_{1}\right)-T_{2}\left(G_{1}\right)=\Pi_{3}\left(G_{2}\right)-T_{2}\left(G_{2}\right)$.

Consider two 1-thorns $a b c$ and $a b d$ where $\operatorname{deg}(a) \geq 2$ and $\operatorname{deg}(c)=\operatorname{deg}(d)=1$, then swapping $a b c$ and $a b d$ gives a $P_{3}$-isomorphism, which we call a $B$-swap.

Suppose abcde is a $P_{5}$ in $G$ such that both $a b c$ and $c d e$ are terminal 1-thorns, i.e., $\operatorname{deg}(a)=\operatorname{deg}(e)=1$ and $\operatorname{deg}(c)=2$, then swapping $a b c$ and $c d e$ gives a $P_{3}$-isomorphism, which we call an $S$-swap.

For distinct $a, b \in V(G)$, let $D_{a, b}$ denote the subgraph of $G$ consisting of the union of all $P_{3}$ 's with ends $a$ and $b$ and with middle vertex of degree 2 in $G$. If $D_{a, b}$ is nonempty we call it a diamond with ends $a$ and $b$. We usually write $V\left(D_{a, b}\right)-\{a, b\}$ as $\left\{c_{1}, c_{2}, \cdots, c_{k}\right\}$ and call $k$ the width of $D_{a, b}$, and refer to $D_{a, b}$ as a $k$-diamond. Note that if $a$ and $b$ are adjacent, the edge $a b$ is not included in $D_{a, b}$. To distinguish the two possibilities, we say that the diamond $D_{a, b}$ is braced if $a$ and $b$ are adjacent in $G$ and unbraced otherwise. For $1 \leq i<j \leq k$, the $P_{3}$ 's $a c_{i} b$ are called diamond paths while the pair of $P_{3}$ 's $c_{i} a c_{j}$ and $c_{i} b c_{j}$ is called a diamond pair. Then swapping $c_{i} a c_{j}$ and $c_{i} b c_{j}$ gives a $P_{3}$-isomorphism, which we call a $D$-swap.

Suppose $\tau_{1}$ and $\tau_{2}$ are $P_{3}$-isomorphisms from $G$ to $H$. We say that $\tau_{1}$ and $\tau_{2}$ are $B$ related if $\tau_{2}^{-1} \circ \tau_{1}$ is the identity or a composition of $B$-swaps. The $S$-related and $D$-related are defined similarly. We use joins of these four equivalence relations: for example, two 
$P_{3}$-isomorphisms are $T B S D$-related if we can get from one to the other by a chain of zero or more $T$-, $B$-, $S$ - and/or $D$-relations.

The following is the main result of [1], based on which we shall solve our problem by case analysis.

Theorem 2.1 Let $\tau$ be a $P_{3}$-isomorphism from $G$ to $H$ such that at least one of $G$ or $H$ is connected. Then $\tau$ is one of the following:

(i) T-related to a $P_{3}$-isomorphism of generalized $K_{3,3}$ type;

(ii) of special Whitney type;

(iii) D-related to a $P_{3}$-isomorphism of Whitney type 3, 4, 5 or 6;

(iv) D-related to a $P_{3}$-isomorphism of bipartite type; or

(v) TBSD-related to an induced $P_{3}$-isomorphism.

The definition for each of the above types will be given in the successive subsections.

For solving our problem, in Theorem 2.1 we only need to consider that the original graphs $G$ and $H$ are nonisomorphic connected graphs with $T_{2}(G)=T_{2}(H)=\emptyset$. Below, we will analyze the types in Theorem 2.1 case by case in detail.

\subsection{Generalized $K_{3,3}$ type}

First, we introduce the following notation which is used in the definition of generalized $K_{3,3}$ type. We write $(c, d) a b(e, f) \mapsto u v w x u$ if $G$ contains the edges $a b, a c, a d, b e, b f, H$ contains the $C_{4} u v w x u$, and $\tau$ maps $c a b \mapsto x u v, d a b \mapsto v w x$, abe $\mapsto u v w$ and $a b f \mapsto w x u$. We also write $a b c(d, e) \mapsto u v w x y$ if $G$ contains the edges $a b, b c, c d, c e, H$ contains the $P_{5} u v w x y$, and $\tau$ maps $a b c \mapsto v w x, b c d \mapsto u v w$ and $b c e \mapsto w x y$. This notation will be reversed (e.g., abcda $\mapsto(w, x) u v(y, z))$ as needed. Then, define the generalized $K_{3,3}$ type as follows:

Either $\tau$ or $\tau^{-1}$ as in the following cases (i) through (vii), or any equivalent $P_{3^{-}}$ isomorphism, is said to be of generalized $K_{3,3}$ type.

(i) $(c, d) a b(e, f) \mapsto u_{1} v_{1} u_{2} v_{2} u_{1}$, and $c a d$ and ebf map to $P_{3}$ components of $H$.

(ii) $(c, d) a b(e, f) \mapsto u_{1} v_{1} u_{2} v_{2} u_{1}, k e b f h \mapsto y v_{3} u_{1}\left(v_{1}, v_{2}\right)$, and cad maps to a $P_{3}$ component.

(iii) $(c, d) a b(e, f) \mapsto u_{1} v_{1} u_{2} v_{2} u_{1},(k, l) e b(a, f) \mapsto u_{1} v_{1} u_{2} v_{3} u_{1},(h, i) f b(a, e) \mapsto u_{1} v_{2} u_{2} v_{3} u_{1}$, and cad, kel and hfi map to $P_{3}$ components.

(iv) $(c, d) a b(e, f) \mapsto u_{1} v_{1} u_{2} v_{2} u_{1}$, ecadg $\mapsto x u_{3} v_{1}\left(u_{1}, u_{2}\right)$, and cebfh $\mapsto y v_{3} u_{1}\left(v_{1}, v_{2}\right)$. Note that $G$ and $H$ are connected and isomorphic.

(v) $(c, d) a b(e, f) \mapsto u_{1} v_{1} u_{2} v_{2} u_{1}$, ebfhe $\mapsto\left(v_{1}, v_{2}\right) u_{1} v_{3}(y, z)$, and cad maps to yv $v_{3} z$. Again $G$ and $H$ are connected and isomorphic.

(vi) $(c, d) a b(e, f) \mapsto u_{1} v_{1} u_{2} v_{2} u_{1},(c, d) e b(a, f) \mapsto u_{1} v_{1} u_{2} v_{3} u_{1},(h, i) f b(a, e) \mapsto u_{1} v_{2} u_{2} v_{3} u_{1}$, aceda $\mapsto(w, x) u_{3} v_{1}\left(u_{1}, u_{2}\right)$, and $h f i$ maps to $w u_{3} x$. Again $G$ and $H$ are connected and isomorphic. 
(vii) The construction on $K_{3,3} ; G \cong H \cong K_{3,3}$.

Remark 1. For generalized $K_{3,3}$ type, it is easy to get the following results:

1. For cases (i), (ii) and (iii), $G$ and $H$ are nonisomorphic, but $H$ is not connected and there are isolated vertices in $P_{3}(G)$ and $P_{3}(H)$.

2. For cases (iv) and (vii), $G$ and $H$ are connected with $T_{2}(G)=T_{2}(H)=\emptyset$, but $G$ and $H$ are isomorphic.

3. For cases (v) and (vi), $G$ and $H$ are connected, but are isomorphic and there are isolated vertices in $P_{3}(G)$ and $P_{3}(H)$.

Thus there is no pair of nonisomorphic connected graphs with isomorphic connected $P_{3^{-}}$ graphs in generalized $K_{3,3}$ type.

\subsection{Special Whitney type}

Let $S W$ be the graph obtained by subdividing each edge of $K_{1,3}$ exactly once, then $P_{3}(S W) \cong C_{6}$. Rotation of this $C_{6}$ by one step is a noninduced $P_{3}$-isomorphism from $S W$ to itself, then we say this or any equivalent $P_{3}$-isomorphism is of special Whitney type.

Hence there is also no pair of nonisomorphic connected graphs with isomorphic connected $P_{3}$-graphs by the definition of special Whitney type.

\subsection{Whitney type $3,4,5$ or 6}

In this subsection, we begin with a general idea which will be used here and in the next subsection. Suppose $F$ is a graph. A diamond inflation of $F$ is a graph obtained by replacing each edge $a b \in E(F)$ by an unbraced $s_{a b}$-diamond $D_{a, b}\left(s_{a b} \geq 1\right)$, and adding $t_{a}$ terminal edges incident with each $a \in V(F)\left(t_{a} \geq 0\right)$. Suppose $\varphi$ is an edge-isomorphism between graphs $F$ and $F^{\prime}$, and suppose $I$ and $I^{\prime}$ are diamond inflations of $F$ and $F^{\prime}$, respectively, with the following property: for every $a b \in E(F)$, if $\varphi(a b)=u v$ then (i) $s_{u v}=s_{a b}$ and (ii) $t_{u}+t_{v}=t_{a}+t_{b}$. Obtain $G$ and $H$ from $I$ and $I^{\prime}$, respectively, by adding star components to one of them (if necessary) to make the numbers of 2-thorns equal. Then we can define a $P_{3}$-isomorphism $\tau$ from $G$ to $H$ and say that $\tau$ is a diamond inflation of $\varphi$.

Remark 2. If $D_{a, b}$ is a nontrivial diamond (i.e., $s_{a b}>1$ ) in $G$, then there exists a unique and nontrivial diamond $D_{u, v}$ in $H$ (see the proof in [1]).

The type in this subsection is related to Whitney's exceptional edge-isomorphisms which is stated as follows:

Theorem 2.2 (Whitney [6]) Suppose that $\varphi$ is an edge-isomorphism from $G$ to $H$ where $G$ and $H$ are both connected. If $\varphi$ is not induced, then $i=|E(G)|=|E(H)| \in$ $\{3,4,5,6\}, G$ and $H$ are isomorphic to $W_{i}$ and $W_{i}^{\prime}$ in some order, and $\varphi$ is equivalent to $\varphi_{i}$ or $\varphi_{i}^{-1}$, where 
(i) $W_{6} \cong W_{6}^{\prime} \cong K_{4}$, with $V\left(W_{6}\right)=\{a, b, c, d\}, V\left(W_{6}^{\prime}\right)=\{u, v, w, x\}$, and $\varphi_{6}$ maps $a b \mapsto u v, a c \mapsto u w, a d \mapsto v w, b c \mapsto u x, b d \mapsto v x$ and $c d \mapsto w x ;$

(ii) $W_{5}=W_{6}-c d, W_{5}^{\prime}=W_{6}^{\prime}-w x$ and $\varphi_{5}=\varphi_{6} \mid E\left(W_{5}\right)$;

(iii) $W_{4}=W_{6}-\{b d, c d\}, W_{4}^{\prime}=W_{6}^{\prime}-\{v x, w x\}$ and $\varphi_{4}=\varphi_{6} \mid E\left(W_{4}\right)$; and

(iv) $W_{3}=W_{6}-\{b c, b d, c d\} \cong K_{1,3}, W_{3}^{\prime}=W_{6}^{\prime}-x \cong K_{3}$, and $\varphi_{3}=\varphi_{6} \mid E\left(W_{3}\right)$.

Then a $P_{3}$-isomorphism $\tau$ is said to be of Whitney type $i$ if $\tau$ or $\tau^{-1}$ is equivalent to a diamond inflation of $\varphi_{i}$ as above for $i=3,4,5,6$.

Denote by $t_{z}$ the number of terminal edges incident with $z$ for $z$ in $\{a, b, c, d\}$ or $\{u, v, w, x\}$. Whitney type $P_{3}$-isomorphisms, according to condition (ii) of Diamond Inflation, give one equation from each pair of corresponding edges of the original Whitney graphs. In all four types, the corresponding edges $a b$ and $u v, a c$ and $u w, a d$ and $v w$ give that $t_{a}+t_{b}=t_{u}+t_{v}, t_{a}+t_{c}=t_{u}+t_{w}, t_{a}+t_{d}=t_{v}+t_{w}$, respectively. Solving for $t_{u}, t_{v}$, $t_{w}$ in terms of $t_{a}, t_{b}, t_{c}, t_{d}$ we get

$$
\left\{\begin{array}{l}
t_{u}=\frac{1}{2}\left(t_{a}+t_{b}+t_{c}-t_{d}\right) \\
t_{v}=\frac{1}{2}\left(t_{a}+t_{b}-t_{c}+t_{d}\right) \\
t_{w}=\frac{1}{2}\left(t_{a}-t_{b}+t_{c}+t_{d}\right)
\end{array}\right.
$$

In Whitney type 4,5 or 6 the corresponding edges $b c$ and $u x$ give that $t_{b}+t_{c}=t_{u}+t_{x}$. Combining Equ.(1), we obtain

$$
t_{x}=\frac{1}{2}\left(-t_{a}+t_{b}+t_{c}+t_{d}\right)
$$

We assume that $t_{x}=0$ in Whitney type 3 as $x \notin V\left(W_{3}^{\prime}\right)$. Because we require connected $P_{3}$-graphs, in the above Equ.(1) and Equ.(2) we must have $t_{z}=0$ or 1 for every $z \in$ $\{a, b, c, d\} \cup\{u, v, w, x\}$. We write $\left(t_{a}, t_{b}, t_{c}, t_{d}\right) \mapsto\left(t_{u}, t_{v}, t_{w}, t_{x}\right)$ if $t_{a}, t_{b}, t_{c}, t_{d}=0$ or 1 , the corresponding solutions for $t_{u}, t_{v}, t_{w}, t_{x}$ by Equ.(1) and Equ.(2). For example: $(1,0,0,1) \mapsto(0,1,1,0)$ denotes that $t_{a}=1, t_{b}=t_{c}=0$ and $t_{d}=1$ correspond to solutions $t_{u}=0$ and $t_{v}=t_{w}=1$ by Equ.(1) and $t_{x}=0$ by Equ.(2). So it is easy to check that there are only the following eight cases satisfying $t_{z}=0$ or 1 for every $z \in\{a, b, c, d\} \cup\{u, v, w, x\}$ :

(i) $(0,0,0,0) \mapsto(0,0,0,0)$ for all four types.

(ii) $(1,1,0,0) \mapsto(1,1,0,0)$ for all four types.

(iii) $(1,0,1,0) \mapsto(1,0,1,0)$ for all four types.

(iv) $(1,0,0,1) \mapsto(0,1,1,0)$ for all four types.

(v) $(0,1,1,0) \mapsto(1,0,0,0)$ for type 3 and $(0,1,1,0) \mapsto(1,0,0,1)$ for type 4,5 or 6 .

(vi) $(0,1,0,1) \mapsto(0,1,0,0)$ for type 3 and $(0,1,0,1) \mapsto(0,1,0,1)$ for type 4,5 or 6 .

(vii) $(0,0,1,1) \mapsto(0,0,1,0)$ for type 3 and $(0,0,1,1) \mapsto(0,0,1,1)$ for type 4,5 or 6 .

(viii) $(1,1,1,1) \mapsto(1,1,1,0)$ for type 3 and $(1,1,1,1) \mapsto(1,1,1,1)$ for type 4,5 or 6 . 
If a $P_{3}$-isomorphism $\tau$ or $\tau^{-1}$ is equivalent to a diamond inflation of $\varphi_{i}$ as above, and falls into one of the above cases (i) through (viii), then $\tau$ is said to be of special Whitney type $i$ for $i=3,4,5$ or 6 .

Let $\tau$ be a $P_{3}$-isomorphism from $G$ to $H$. It is well-known that $K_{1,3}$ and $K_{3}$ are the only pair of nonisomorphic connected graphs with the same line graph. So, if $\tau$ is of special Whitney type 3 , then $G \neq H$. If $\tau$ is of special Whitney type $i(i=4,5$ or 6$)$, in order to find pairs of nonisomorphic connected graphs with isomorphic $P_{3}$-graphs, we should choose suitable widths of each corresponding diamonds. Otherwise, for example, let $G$ and $H$ be diamond inflations of $W_{4}$ and $W_{4}^{\prime}$, respectively, with $s_{e}=2$ and $t_{z}=1$ for every $e \in E\left(W_{4}\right) \cup E\left(W_{4}^{\prime}\right)$ and every $z \in\{a, b, c, d\} \cup\{u, v, w, x\}$. Obviously, $G \cong H$.

\subsection{Bipartite type}

First, we also introduce the definition of bipartite type. Start with a positive integer $k$ and an arbitrary bipartite graph $F$ with at least one edge and with a bipartition $(A, B)$. Let $I$ and $I^{\prime}$ be different diamond inflations of $F$, where each edge $e$ is inflated to a diamond of the same width $s_{e}$ both times, but in producing $I$ each vertex $v$ has $t_{v}$ terminal edges added, while in producing $I^{\prime}$ it has $t_{v}^{\prime}$ terminal edges added. where

$$
t_{v}^{\prime}= \begin{cases}t_{v}-k & \text { if } v \in A \\ t_{v}+k & \text { if } v \in B\end{cases}
$$

Thus, we need $t_{v} \geq k$ for all $v \in A$. Let $\varphi$ be the identity edge-isomorphism from $F$ to itself. Clearly $\varphi, I$ and $I^{\prime}$ satisfy condition (i) of Diamond Inflation, and condition (ii) is satisfied because each edge of $F$ has the form $a b$ with $a \in A$ and $b \in B$, so that $t_{a}^{\prime}+t_{b}^{\prime}=\left(t_{a}-k\right)+\left(t_{b}+k\right)=t_{a}+t_{b}$. We can therefore obtain a $P_{3}$-isomorphism $\tau$ by diamond inflation; $\tau$ is in general not induced. We say $\tau$ and $\tau^{-1}$, or any equivalent $P_{3}$-isomorphisms, are of bipartite type.

This case is similar to the above Whitney type. Because we require that the $P_{3}$-graphs of $I$ and $I^{\prime}$ are connected, we must have $t_{v}, t_{v}^{\prime}=0$ or 1 for every $v \in A \cup B$. Since $k \leq t_{v}(v \in A)$, we have $k=0$ or 1 . If $k=0$, then $I \cong I^{\prime}$. If $k=1$, then $t_{u}=1$ for all $u \in A$ and $t_{v}=0$ for all $v \in B$. Otherwise, if there is a vertex $u_{0} \in A$ with $t_{u_{0}}=0$ or a vertex $v_{0} \in B$ with $t_{v_{0}}=1$, then $t_{u_{0}}^{\prime}=-1$ or $t_{v_{0}}^{\prime}=2$ by Equ.(3). Therefore we have a $P_{3}$-isomorphism $\tau_{0}$ from $I$ to $I^{\prime}$, where $t_{u}=1$ and $t_{u}^{\prime}=0$ for all $u \in A, t_{v}=0$ and $t_{v}^{\prime}=1$ for all $v \in B$, respectively. Then we say that $\tau_{0}$ and $\tau_{0}^{-1}$, or any equivalent $P_{3}$-isomorphism, are of special bipartite type. Therefore, this is the only case to find pairs of nonisomorphic connected graphs which have isomorphic connected $P_{3}$-graphs in the bipartite type.

\subsection{TBSD-related to an induced $P_{3}$-isomorphism}

In this subsection, we require that there are no isolated vertices in $P_{3}$-graphs. Then all $P_{3}$-isomorphisms are $B S D$-related to an induced one. It is clear that if two original graphs 
$G$ and $H$ are connected with an isomorphic $P_{3}$-graph, then $G \cong H$ by the definition of $B S D$-related. Thus in this type, if we require connected $P_{3}$-graphs, then the original graph and its $P_{3}$-graph are one to one.

From the arguments in the above five subsections, we get the following corollary which is essential to the solution of our problem.

Corollary 2.3 Let $\tau$ be a $P_{3}$-isomorphism from $G$ to $H$, where $G$ and $H$ are nonisomorphic connected graphs with an isomorphic connected $P_{3}$-graph. Then $\tau$ is one of the following:

(i) D-related to a $P_{3}$-isomorphism of special Whitney type 3, 4, 5 or 6; or

(ii) D-related to a $P_{3}$-isomorphism of special bipartite type.

\section{Main result}

Now we can state and show the main result of this paper.

Theorem 3.1 There is no triple of mutually nonisomorphic connected graphs with an isomorphic connected $P_{3}$-graph.

Proof. Assume, to the contrary, that there exists a triple of mutually nonisomorphic connected graphs $G_{1}, G_{2}$ and $G_{3}$ which have an isomorphic connected $P_{3}$-graph. Let $\tau_{i}$ be a $P_{3}$-isomorphism from $G_{i}$ to $G_{i+1}$, then $\tau_{i}$ will be one of two types in Corollary 2.3 for $i=1,2$.

Case 1. $\tau_{1}$ and $\tau_{2}$ are of the same type.

Subcase $1.1 \tau_{1}$ and $\tau_{2}$ are both of $D$-related to a $P_{3}$-isomorphism of special Whitney type $i$ for $i=3,4,5$ or 6 .

By the definition of $\tau_{1}$, let $G_{1}$ and $G_{2}$ be diamond inflations of $W_{i}$ and $W_{i}^{\prime}$, respectively, with one of the eight cases in subsection 2.3. For $i=3$, without loss of generality, suppose that $t_{a}=1, t_{b}=t_{c}=0, t_{d}=1, t_{u}=0, t_{v}=t_{w}=1$ and $t_{x}=0$. Since $\tau_{1}$ and $\tau_{2}$ are of the same type, $G_{3}$ is also a diamond inflation of $W_{3}$, and $t_{a}=1, t_{b}=t_{c}=0, t_{d}=1$ by Equ.(1). Hence $G_{1} \cong G_{3}$, a contradiction. For $i=4,5$ or 6 , by a similar argument as the case for $i=3$, we also get $G_{1} \cong G_{3}$, a contradiction.

Subcase $1.2 \tau_{1}$ and $\tau_{2}$ are both of $D$-related to a $P_{3}$-isomorphism of special bipartite type.

This subcase is similar to Subcase 1.1. Denote by $F$ an arbitrary bipartite graph with a bipartition $(A, B)$. Then assume that $G_{1}$ and $G_{2}$ are different diamond inflations of $F$, respectively, where $t_{u}=1$ for all $u \in A$ and $t_{v}=0$ for all $v \in B$ in $G_{1} ; t_{u}=0$ for all $u \in A$ and $t_{v}=1$ for all $v \in B$ in $G_{2}$. Thus we can easily obtain that $G_{3}$ is also a diamond inflation of $F$ with $t_{u}=1$ for all $u \in A$ and $t_{v}=0$ for all $v \in B$ in $G_{3}$ by the definition of $\tau_{2}$. Then $G_{1} \cong G_{3}$, contrary to the assumption.

Case 2. $\tau_{1}$ and $\tau_{2}$ are of different types.

Let $\tau_{1}$ be $D$-related to a $P_{3}$-isomorphism of special Whitney type $i$ for $i=3,4,5$ or 6 and $\tau_{2}$ be $D$-related to a $P_{3}$-isomorphism of special bipartite type. 
For $i=4,5$ or 6 , by the definition of $\tau_{1}, G_{1}$ and $G_{2}$ are diamond inflations of $W_{i}$ and $W_{i}^{\prime}$ which have odd cycles; and also by $\tau_{2}, G_{2}$ and $G_{3}$ are different diamond inflations of some bipartite graph. Then $\tau_{1}$ must be $D$-related to a $P_{3}$-isomorphism of special Whitney type 3 . Thus there is only one possibility: $G_{2}$ is a diamond inflation of $K_{1,3}$, where $K_{1,3}$ has a bipartition $A=\{a\}, B=\{b, c, d\}$. By the definition of special Whitney type $3, \tau_{1}$ falls into one of the following eight cases: $(0,0,0,0) \mapsto(0,0,0,0),(1,1,0,0) \mapsto(1,1,0,0)$, $(1,0,1,0) \mapsto(1,0,1,0),(1,0,0,1) \mapsto(0,1,1,0),(0,1,1,0) \mapsto(1,0,0,0),(0,1,0,1) \mapsto$ $(0,1,0,0),(0,0,1,1) \mapsto(0,0,1,0)$, or $(1,1,1,1) \mapsto(1,1,1,0)$. However, by the definition of special bipartite type, there are only two choices: either $t_{a}=0, t_{b}=t_{c}=t_{d}=1$, or $t_{a}=1, t_{b}=t_{c}=t_{d}=0$. Finally, there does not exist any graph $G_{2}$ that has common property of two different types at the same time. So $\tau_{1}$ and $\tau_{2}$ must be of the same type, a contradiction. The proof is thus complete.

Acknowledgement. The authors are very grateful to the referees for their valuable suggestions and comments, which helped to improve the presentation of the paper.

\section{References}

[1] R.E.L. Aldred, M.N. Ellingham, R.L. Hemminger and P. Jipsen, $P_{3}$-isomorphisms for graphs, J. Graph Theory 26 (1997) 35-51.

[2] J.A. Bondy and U.S.R. Murty, Graph Theory with Applications, MacMillan/Elesevier, London/New York, 1976.

[3] H.J. Broersma and C. Hoede, Path graphs, J. Graph Theory 13 (1989) 427-444.

[4] R.L. Hemminger and L.W. Beineke, Line graphs and line digraphs, in: L.W. Beineke and R.J. Wilson, eds, Selected Topics in Graph Theory, Academic Press, London, New York, San Francisco, 1978.

[5] X. Li and B. Zhao, Isomorphisms of $P_{k}$-graphs for $k \geq 4$, Discrete Math. 289 (2004) 71-80.

[6] H. Whitney, Congruent graphs and connectivity of graphs, Amer. J. Math. 54 (1932) $150-168$. 